\title{
ADUBAÇÃO ORGÂNICA DA BATATA COM ESTERCO E, OU, Crotalaria juncea. II - DISPONIBILIDADE DE N, P E K NO SOLO AO LONGO DO CICLO DE CULTIVO ${ }^{(1)}$
}

\author{
Tácio Oliveira da Silva ${ }^{(2)} \&$ Rômulo Simões Cezar Menezes ${ }^{(3)}$
}

\begin{abstract}
RESUMO
Os solos do Agreste paraibano têm baixa fertilidade e a prática usual de adubação é a incorporação de esterco na época do plantio. Entretanto, dependendo da qualidade do esterco, essa prática pode causar a imobilização de nutrientes do solo durante os primeiros meses de cultivo. É possível que o cultivo e incorporação de Crotalaria juncea, combinado com o esterco, possa promover mineralização mais sincronizada com a demanda de nutrientes pelas plantas. No presente estudo, realizado em 2003, foram conduzidos experimentos de campo e em casa de vegetação para testar essa hipótese. As parcelas de campo foram previamente cultivadas com batata no período de 1996 a 2002 e submetidas anualmente aos seguintes tratamentos: plantio e incorporação da crotalária na época da floração (C); adição de $15 \mathrm{t} \mathrm{ha}^{-1}$ de esterco (E); plantio e incorporação de crotalária $+7,5 \mathrm{t} \mathrm{ha}^{-1}$ de esterco (CE); e testemunha, sem esterco ou crotalária (T). Em 2003, foram aplicados os mesmos tratamentos de adubação orgânica e foi avaliada a dinâmica da decomposição e liberação de nutrientes pelo material vegetal e esterco contidos em sacolas de náilon incorporadas ao solo das parcelas de campo. Também se avaliou a dinâmica da disponibilidade de N, P e K no solo das parcelas de campo, por meio de coletas periódicas de solo. No ensaio em casa de vegetação, foram realizados cultivos sucessivos de capim-buffel (Cenchrus ciliaris L.) por um período de 300 dias, utilizando-se amostras do solo das parcelas de campo. As perdas de massa e nutrientes do material incorporado ao solo foram maiores nos primeiros 30 dias da incubação, em todos os tratamentos. No final do ensaio, as proporções
\end{abstract}

\footnotetext{
(1) Parte da Tese de Mestrado do primeiro autor apresentada à Universidade Federal de Pernambuco - UFPE. Trabalho financiado pelo CNPq, CAPES e pelo Interamerican Institute for Global Change Research-IAI (CRN 001). Recebido para publicação em março de 2005 e aprovado em janeiro de 2007.

(2) Doutorando do Programa de Solos e Nutrição de Plantas, Departamento de Ciência do Solo, Universidade Federal de Lavras - UFLA. Caixa Postal 3037, CEP 37200-000 Lavras (MG). E-mail: taccios@bol.com.br

(3) Professor Adjunto do Departamento de Energia Nuclear, Universidade Federal de Pernambuco - UFPE. Av. Prof. Luís Freire 1000, CEP 50740-540 Recife (PE). Bolsista do CNPq. E-mail: rmenezes@ufpe.br
} 
de matéria seca e nutrientes remanescentes foram maiores nos tratamentos $\mathrm{E}$ CE que nos tratamentos $\mathrm{C}$ e $\mathrm{T}$. No solo das parcelas de campo, o tratamento $\mathrm{E}$ aumentou os teores de $\mathrm{P}$ e $\mathrm{K}$ extraíveis (Mehlich-1) do solo ao longo do período do estudo, porém provocou a imobilização de $\mathrm{N}$ do solo nas primeiras semanas. $\mathrm{O}$ tratamento $\mathrm{C}$ aumentou o teor de $\mathrm{N}$ mineral do solo no período imediatamente após a incorporação, mas não aumentou o teor de $\mathbf{P}$ e $\mathrm{K}$ extraíveis. No ensaio em casa de vegetação, o capim-buffel acumulou mais biomassa aérea e nutrientes no primeiro corte, 35 dias após o transplantio do capim, no solo do tratamento CE. Nos cortes subseqüentes, o tratamento E levou à maior produção de biomassa $\mathrm{e}$ acumulação de nutrientes, indicando que a limitação de $\mathrm{N}$ no período inicial após a incorporação de esterco ao solo prejudicou o crescimento do buffel. Os resultados corroboram a hipótese de que o plantio e incorporação da crotalária, combinado com a aplicação de apenas a metade da dose usual de esterco caprino, promoveu mineralização de nutrientes mais sincronizada com as necessidades das culturas agrícolas, pois foi capaz de evitar a imobilização de $\mathrm{N}$ do solo no período inicial de cultivo e elevou os teores de $\mathrm{P}$ e $\mathrm{K}$ disponíveis ao longo de todo o período.

Termos de indexação: Neossolo Regolítico, região semi-árida, adubação verde, nitrogênio, fósforo, potássio.

\section{SUMMARY: ORGANIC FERTILIZATION OF POTATO WITH MANURE AND, OR, Crotalaria juncea. II - SOIL N, P, AND K AVAILABILITY THROUGHOUT THE GROWING SEASON}

Soil fertility levels in the Agreste region, state of Paraíba, Brazil are generally low. Usually, animal manure is applied to soils to supply agricultural crops with nutrients. However, depending on the quality of the manure applied, incorporation can lead to soil nitrogen immobilization in the beginning of the growing season. It was hypothesized that planting and incorporating crotalaria (Crotalaria juncea) together with animal manure could synchronize the nutrient mineralization pattern more specifically with crop demand. In 2003, field and green-house experiments were carried out to test this hypothesis. Prior to the current study, the field plots were cultivated with potato from 1996 to 2002 and subjected annually to the following organic fertilization treatments: planting and incorporation of crotalaria during flowering (C); manure incorporation, $15 \mathrm{t} \mathrm{ha} \mathrm{h}^{-1}(\mathrm{E})$; planting and incorporation of crotalaria $+7.5 t \mathrm{ha}^{-1}$ manure (CE); and control plots (T). During 2003, these treatments were repeated and the decomposition dynamics evaluated in samples contained in $0.5 \mathrm{~mm}$-mesh nylon decomposition bags with organic materials buried at $20 \mathrm{~cm}$ soil depth. Soil nutrient availability was also evaluated in soil samples $(0-20 \mathrm{~cm})$ during the growing season. In the green-house, buffel grass (Cenchrus ciliaris L.) was grown for 300 days in pots containing soil from the field plots and submitted to successive cuts of the shoots. Dry matter and nutrient losses from the incorporated material in the decomposition bags peaked in the first 30 days in all treatments. At the end of the growing season, dry matter and nutrient losses were lower for the treatments with manure, compared to treatments $C$ and T. Manure incorporation increased soil $P$ and $K$ levels throughout the study period, but led to net $N$ immobilization during the first few weeks after incorporation. Treatment $C$ increased soil mineral $N$ concentration shortly after incorporation, but decreased later in the growing season. Soil $P$ and $K$ levels remained unchanged. In the green-house study, 35 days after planting the biomass accumulation and nutrient uptake by buffel grass was the highest in treatment $C E$. In the following harvests, treatment $E$ led to a greater biomass and nutrient uptake by buffel grass, indicating that $N$ immobilization after manure incorporation limited plant growth in the first weeks. These results show that planting and incorporation of crotalaria coupled with the application of only half the usual manure rate was enough to avoid soil net $N$ immobilization and increased soil $P$ and $K$ levels. The combined application of animal and green manure leads to availability of soil nutrients more synchronized with the plant demand.

Index terms: Entisol, semi-arid region, green manure, nitrogen, phosphorus, potassium. 


\section{INTRODUÇÃO}

A baixa fertilidade dos solos é um dos principais fatores limitantes da produtividade nas propriedades agrícolas familiares no Agreste da Paraíba (Menezes et al., 2002). Nessa região, destaca-se a agricultura de sequeiro com culturas anuais de subsistência, com baixas produtividades, decorrentes das irregularidades climáticas e do manejo inadequado do solo (Nascimento et al., 2003). Em geral, nos solos do semi-árido nordestino, $\mathrm{N}$ e $\mathrm{P}$ encontram-se, em baixa disponibilidade, o que limita significativamente a produtividade vegetal (Sampaio et al., 1995). O uso de fertilizantes químicos nitrogenados e fosfatados é bastante reduzido, devido ao seu alto custo e ao baixo poder aquisitivo da maioria dos agricultores, bem como ao risco proporcionado pela variabilidade do regime de chuvas. Por esse motivo, nessa região, o manejo da fertilidade do solo depende principalmente do manejo da matéria orgânica (Tiessen et al., 1994; Nascimento et al., 2003).

A incorporação do esterco ao solo é uma alternativa amplamente adotada para suprimento de nutrientes nos solos da região semi-árida. No entanto, a reduzida disponibilidade de esterco nas propriedades leva grande parte dos agricultores a importá-lo de regiões circunvizinhas, o que eleva os custos de produção (Menezes et al., 2002). Outra limitação quanto ao uso do esterco de curral é que este - principalmente em anos de reduzida precipitação pluviométrica - pode prejudicar as culturas agrícolas devido à incompleta decomposição e à imobilização de nutrientes do solo (Holanda, 1990; Palm et al., 2001).

Uma das alternativas para minimizar as limitações do uso do esterco é sua utilização consorciada com adubação verde com leguminosas. A adubação verde pode aumentar o conteúdo de matéria orgânica do solo, melhorar as condições físicas, químicas e biológicas deste e favorecer o crescimento e rendimento dos cultivos de importância econômica (Monegat, 1991). As leguminosas têm geralmente sido mais usadas como adubo verde devido à sua rusticidade, à elevada produção de matéria seca, ao sistema radicular profundo e à simbiose com bactérias fixadoras do $\mathrm{N}_{2}$ atmosférico. Dentre as leguminosas utilizadas na adubação verde e cobertura do solo, destaca-se Crotalaria juncea L., por ser uma planta pouco exigente em nutrientes, com grande potencial de fixação biológica de $\mathrm{N}_{2}$, e por apresentar crescimento rápido (Araújo \& Almeida, 1993).

As leguminosas apresentam fitomassa bastante lábil, que favorece a redução da relação $\mathrm{C} / \mathrm{N}$, aumentando a atividade microbiana - conseqüentemente, a mineralização do material vegetal - e disponibilizando o N, evitando a imobilização deste no solo pela microbiota (Palm et al., 2001). Uma das limitações da adubação verde, por meio do cultivo de cobertura com leguminosas e sua incorporação ao solo, é que, com exceção do N, a incorporação da biomassa da leguminosa não repõe os nutrientes retirados do solo pelas culturas comerciais (Sampaio \& Maluf, 1999; Silva, 2004).

Portanto, é possível que a aplicação combinada de adubo verde e esterco possa minimizar a deficiência de $\mathrm{N}$ do solo, em razão da labilidade do adubo verde, e prover ao solo $\mathrm{P}$ e outros nutrientes contidos no esterco. Com isso, espera-se promover maior sincronização entre a mineralização e a demanda de nutrientes pelas culturas, aumentando a produtividade e contribuindo para a redução dos custos de produção nos sistemas agrícolas familiares no semi-árido (Menezes et al., 2002).

Silva et al. (2007) realizaram um experimento de campo no período de 1996 a 2002 para avaliar o efeito acumulado da incorporação de esterco e, ou, crotalária sobre o estoque de nutrientes do solo e a produtividade de tubérculos de batata (Solanum tuberosum L). Esses autores observaram que a incorporação de $15 \mathrm{t} \mathrm{ha}^{-1}$ de esterco forneceu maior quantidade de nutrientes ao solo, mas a produtividade de tubérculos foi maior com a incorporação de $7,5 \mathrm{t} \mathrm{ha}^{-1}$ de esterco combinada com a incorporação de crotalária. Foi sugerida a hipótese de que, provavelmente, isso ocorreu devido à melhor sincronização entre a disponibilidade de nutrientes do solo e a demanda da batata durante o período de cultivo. O presente trabalho foi realizado para testar essa hipótese. Assim, em 2003 foram realizados três experimentos para avaliar a dinâmica de nutrientes do solo após a incorporação de esterco e, ou, crotalária. O objetivo desses experimentos foi quantificar, ao longo do período de cultivo: as perdas de massa e nutrientes pelo esterco e, ou, crotalária incorporados ao solo em parcelas de campo; periodicamente o teor de nutrientes disponíveis no solo das parcelas de campo; e a produção de massa seca e acúmulo de nutrientes pelo capim-buffel, ao longo de 300 dias de cultivo em casa de vegetação, utilizandose o solo das parcelas de campo.

\section{MATERIAL E MÉTODOS}

\section{Descrição da área do estudo de campo}

O estudo de campo foi feito durante a estação úmida (fevereiro a agosto) de 2003, em parcelas experimentais localizadas no Centro Agroecológico São Miguel, de propriedade da ONG Assessoria e Serviços a Projetos em Agricultura Alternativa (AS-PTA), no município de Esperança, PB. No período anterior ao deste estudo, entre 1996 e 2002, as parcelas experimentais de campo receberam adubação orgânica anualmente e foram cultivadas com batata. Os tratamentos de adubação orgânica consistiram em: plantio e incorporação da crotalária na época da floração (C); adição de $15 \mathrm{t} \mathrm{ha}^{-1}$ de esterco (E); plantio e incorporação de crotalária + 7,5 t ha ${ }^{-1}$ de esterco (CE); e testemunha, sem esterco ou crotalária (T). Esses tratamentos foram 
implementados anualmente nas mesmas parcelas ao longo de todo o período, de forma que viabilizasse a avaliação do efeito acumulado dos tratamentos. Mais informações sobre o histórico das parcelas experimentais no período de 1996 a 2002 podem ser obtidas em Silva et al. (2007). As características químicas do solo nas parcelas experimentais, para cada um dos tratamentos de adubação orgânica na final do ciclo de cultivo, em 2002, após a colheita dos tubérculos, estão descritas no quadro 1.

A média de precipitação pluvial da região é de cerca de $800 \mathrm{~mm}$ por ano, com regime de chuvas unimodal, sendo a estação úmida geralmente de março a agosto e a seca, de setembro a fevereiro (Sabourin et al., 2000) (Figura 1).

O solo da área experimental é classificado como Neossolo Regolítico de textura franco-arenosa e declividade em torno de $5 \%$. As parcelas experimentais tinham $60 \mathrm{~m}^{2}(6 \times 10 \mathrm{~m})$ e foram distribuídas em blocos casualizados, com quatro repetições.

Em fevereiro de 2003, a crotalária foi plantada logo após as primeiras chuvas nas parcelas dos tratamentos $\mathrm{C}$ e CE. O plantio foi feito em sulcos, no espaçamento de 0,50 x 0,10 m. Simultaneamente ao crescimento da crotalária, nas parcelas dos tratamentos T e E cresciam ervas espontâneas. No momento de plena floração, aproximadamente 50 dias após o plantio, a crotalária e as ervas espontâneas foram cortadas com rolo-faca puxado por animal de tração.

O preparo do solo para formar os leirões consistiu no arraste da camada superficial deste, de forma que preparasse uma pequena leira com aproximadamente $20 \mathrm{~cm}$ de altura, denominada "encama" do leirão (Sabourin et al., 2000). A crotalária e as ervas espontâneas cortadas foram amontoadas, com o auxílio de enxadas, sobre a encama. Nos tratamentos com incorporação de esterco, este também foi colocado sobre a encama. Depois disso, foi retirado solo da área entre

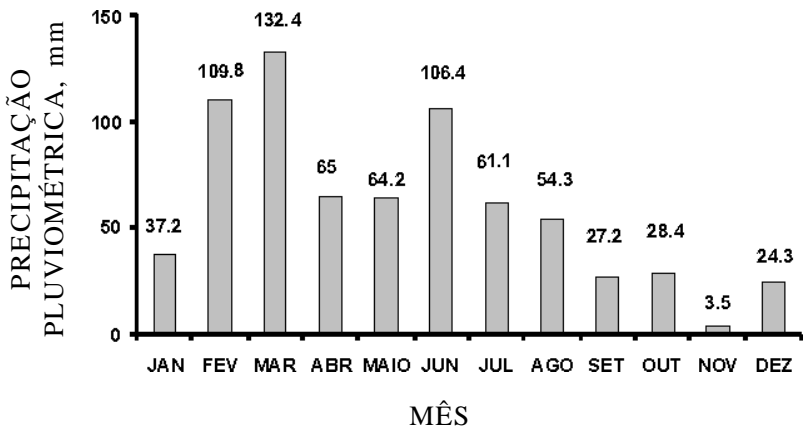

Figura 1. Precipitação pluvial ao longo do ano de 2003 no Centro Agroecológico São Miguel, no município de Esperança, PB.

as encamas e colocado em cima do material vegetal e, ou, esterco, formando leirões com cerca de $40 \mathrm{~cm}$ de altura e $50 \mathrm{~cm}$ de largura na base.

\section{Perdas de massa e nutrientes pelo material incorporado ao solo nas parcelas de campo}

Nas parcelas experimentais de campo foi realizado um experimento para avaliar a dinâmica da decomposição e liberação de nutrientes do esterco e, ou, do material vegetal incorporado ao solo. Para isso, subamostras de esterco, ervas espontâneas e, ou, crotalária foram acondicionadas em bolsas de telas de náilon com dimensões de 10 x $10 \mathrm{~cm}$ e abertura de $0,5 \mathrm{~mm}$. Nos tratamentos C e T o material contido nas bolsas consistiu em subamostras da biomassa da crotalária e das plantas espontâneas, respectivamente, coletadas nas parcelas experimentais de campo antes da incorporação ao solo. Nos tratamentos E e CE, colocaram-se nas bolsas subamostras de esterco e ervas espontâneas, ou esterco e crotalária, respectivamente, para simular o que acontece no campo no momento da adubação orgânica. A quantidade de esterco colocado nas sacolas dos tratamentos E e CE no início do ensaio foi de 25 e $12,5 \mathrm{~g}$, respectivamente. A

Quadro 1. Teor médio de nutrientes e pH do solo em parcelas submetidas à adubação com crotalária e, ou, esterco durante o período de 1996 a $2002^{(1)}$, em um Neossolo Regolítico, na profundidade de 0-20 cm

\begin{tabular}{|c|c|c|c|c|c|c|}
\hline Tratamento( ${ }^{(2)}$ & $\mathrm{N}$ total & P total & $\mathbf{N}$ mineral ${ }^{(3)}$ & P extraível(4) & K extraível & $\mathrm{pH} \mathrm{H}_{2} \mathrm{O}$ \\
\hline $\mathrm{C}$ & 708 & 135 & 32,1 & 9,2 & 85,80 & 6,0 \\
\hline $\mathrm{E}$ & 905 & 181 & 33,2 & 32,1 & 167,70 & 7,3 \\
\hline $\mathrm{CE}$ & 854 & 173 & 28,7 & 22,0 & 175,50 & 7,3 \\
\hline $\mathrm{T}$ & 524 & 125 & 20,9 & 10,0 & 117,0 & 6,7 \\
\hline
\end{tabular}

\footnotetext{
${ }^{(1)}$ Devido à insuficiência de chuvas, as parcelas não foram adubadas e plantadas em 1998 e $1999 .{ }^{(2)} \mathrm{C}=$ plantio e incorporação de Crotalaria juncea antes do plantio da batata; $\mathrm{E}=$ aplicação anual de $15 \mathrm{t} \mathrm{ha}^{-1}$ de esterco de caprinos; $\mathrm{CE}=$ plantio e incorporação de $C$. juncea + aplicação anual de 7,5 $\mathrm{t} \mathrm{ha}^{-1}$ de esterco; $\mathrm{T}=$ testemunha sem adubação. ${ }^{(3)} \mathrm{N}^{-\mathrm{NO}_{3}}{ }^{-}+\mathrm{N}^{-\mathrm{NH}}{ }_{4}^{+}$. ${ }^{(4)} \mathrm{Mehlich}-1$.
} 
quantidade de biomassa da crotalária ou ervas espontâneas foi baseada na produtividade média de biomassa vegetal das parcelas de campo para o ano de 2003 e foi proporcional às doses de esterco. Assim, as bolsas em cada tratamento consistiram de: $25 \mathrm{~g}$ de esterco $+4,86 \mathrm{~g}$ de ervas espontâneas (E); $12,5 \mathrm{~g}$ de esterco + 5,02 g de crotalária (CE); 3,72 g de crotalária (C); e 3,86 g de ervas espontâneas (T). As bolsas foram enterradas no solo dos leirões no momento do plantio da batata, a aproximadamente $20 \mathrm{~cm}$ de profundidade, simulando a mesma profundidade de incorporação dos adubos orgânicos durante o preparo do solo. O delineamento experimental foi em blocos completos ao acaso, com quatro tratamentos e quatro repetições. Ao todo, foram preparadas 96 bolsas (quatro tratamentos $\mathrm{x}$ quatro repetições $\mathrm{x}$ seis datas de amostragem). A primeira amostragem foi realizada sete dias após a incorporação das bolsas ao solo, e as cinco restantes, em intervalos quinzenais, após a primeira amostragem.

Após a coleta, o material vegetal dentro das bolsas foi seco em estufa até massa constante e pesado. Subamostras dos materiais incorporados foram moídas e digeridas, para determinação dos teores de $\mathrm{N}$ e $\mathrm{P}$ (Thomas et al., 1967) e K, por fotometria de chama. Em outras subamostras foi determinado o teor de cinzas do material nas bolsas, para correção do cálculo das perdas de massa e nutrientes do material incorporado, subtraindo-se a massa do solo que aderiu aos materiais colocados dentro das bolsas (Harmon et al., 1999).

\section{Dinâmica da disponibilidade de nutrientes do solo nas parcelas de campo}

Durante o ciclo da batata foram coletadas amostras de solo, nos leirões, na profundidade de 0-20 cm, para analisar a dinâmica do teor de nutrientes. A primeira amostragem foi realizada imediatamente após a incorporação dos adubos orgânicos e o plantio da batata; as demais amostragens foram feitas 7, 25, 62, 76 e 88 dias após a primeira. Foram analisados N$\mathrm{NH}_{4}{ }^{+}$e N-NO ${ }_{3}{ }^{-}$, mediante extração com KCl $1 \mathrm{~mol} \mathrm{~L}^{-1}$ e determinação em auto-analisador Technicon (EPA, 1971), e P e K extraíveis, por Mehlich-1, determinados por colorimetria (Murphy \& Riley, 1962) e por fotometria de chama, respectivamente. $\mathrm{O} \mathrm{pH}$ do solo foi determinado em água por potenciometria, em suspensão na proporção de 1:2,5 (solo:água), de acordo com o método descrito pela Embrapa (1997).

\section{Experimento em casa de vegetação}

Para avaliar o efeito dos tratamentos de adubação orgânica sobre a disponibilidade e absorção de nutrientes pelas plantas, foi realizado um experimento em casa de vegetação, no Departamento de Energia Nuclear da Universidade Federal de Pernambuco, em Recife, durante o período de maio de 2003 a março de 2004, perfazendo um total de 300 dias. Vasos de PVC, de $0,5 \mathrm{dm}^{3}$, com $0,7 \mathrm{~kg}$ de solo, foram plantados com capim-buffel (Cenchrus ciliares). Este capim foi escolhido como planta indicadora por responder bem a diferenças de fertilidade de solo (Fraga, 2002), suportar cortes sucessivos e por ser uma gramínea adaptada às condições da região semi-árida - sobre a qual existem poucas informações disponíveis.

Foi utilizado o solo das parcelas do experimento de campo, coletado após a colheita da batata, em 2002 (Quadro 1). Antes do plantio do capim-buffel, foram incorporados esterco e, ou, crotalária no solo dos respectivos tratamentos, em doses, por vaso, equivalentes às aplicadas no campo. Os cálculos para determinar as doses de cada tratamento foram feitos considerando a massa de solo e a produtividade da biomassa vegetal nas parcelas de campo dos respectivos tratamentos, em 2003. Assim, as doses consistiram de: 7,19 g de esterco + $1,94 \mathrm{~g}$ de ervas espontâneas (E); 3,59 g de esterco + 1,21 g de crotalária (CE); 0,89 g de crotalária (C); e $0,93 \mathrm{~g}$ de ervas espontâneas (T). A composição química do esterco, da crotalária e das ervas espontâneas adicionados ao solo está descrita no quadro 2 . O delineamento experimental utilizado no ensaio foi inteiramente casualizado, com quatro tratamentos e oito repetições.

Quadro 2. Teor médio de N, P e K de amostras de esterco caprino e de ervas espontâneas coletadas em parcelas de campo submetidas a diferentes tratamentos de adubação orgânica

\begin{tabular}{cllll}
\hline Tratamento(1) $^{(1)}$ & Material & $\mathbf{P}$ & $\mathbf{K}$ \\
\hline & & & g kg $^{-1}$ & \\
\cline { 3 - 4 } E & Esterco caprino & 32,35 & 5,48 & 48,03 \\
C & Crotalária & 33,04 & 3,34 & 27,41 \\
CE & Crotalária & 28,90 & 3,98 & 29,53 \\
E & Ervas espontâneas & 25,71 & 4,08 & 36,31 \\
T & Ervas espontâneas & 19,57 & 3,25 & 32,38 \\
\hline
\end{tabular}

(1) $\mathrm{E}=$ aplicação anual de $15 \mathrm{t} \mathrm{ha}^{-1}$ de esterco de caprinos; $\mathrm{C}=$ plantio e incorporação de Crotalaria juncea antes do plantio da batata; $\mathrm{CE}=$ plantio e incorporação de $C$. juncea + aplicação anual de $7,5 \mathrm{t}^{-1}$ de esterco; $\mathrm{T}=$ testemunha sem adubação. 
Inicialmente, o capim-buffel foi semeado em sulcos, em uma bandeja plástica com areia lavada como substrato. Após 10 dias da emergência das plantas, foi feito o transplantio, utilizando quatro plantas por vaso, levando em consideração seu vigor e sua uniformidade. A umidade do solo foi ajustada a $50 \%$ do volume de poros, mediante umedecimento com água deionizada, por meio da pesagem diária dos vasos.

O primeiro corte da biomassa aérea do capim-buffel foi realizado aos 35 dias, e o último, aos 300 dias após o transplantio. Os cortes foram feitos em intervalos de cerca de 40 a 45 dias, dependendo da fenologia das plantas, o que resultou em um total de sete cortes durante o período de 300 dias. Os cortes foram sempre realizados imediatamente antes do início do florescimento das plantas, a uma altura de $2 \mathrm{~cm}$ do colo das raízes. Após o último corte, foram coletadas as raízes, separando-as do solo. Amostras do solo dos vasos, após a colheita, foram coletadas para determinação de $\mathrm{N}-\mathrm{NH}_{4}{ }^{+}, \mathrm{N}-\mathrm{NO}_{3}{ }^{-}, \mathrm{P}$ e K extraíveis e $\mathrm{pH}$, como descrito anteriormente.

A secagem do material vegetal foi realizada em estufa a $60{ }^{\circ} \mathrm{C}$, até alcançar massa constante. Amostras desse material foram moídas em moinho tipo Wiley, digeridas com uma mistura de ácido sulfúrico e água oxigenada; os teores de $\mathrm{N}$ e $\mathrm{P}$ nos extratos da digestão foram analisados por colorimetria (Thomas et al., 1967), e o de K, por fotometria de chama.

As variáveis avaliadas foram matéria seca da parte aérea e das raízes e teor e acúmulo de N, P e K, nos componentes da planta e no solo, após a colheita.

\section{Análise estatística}

Os dados foram analisados estatisticamente por meio da análise de variância e as médias comparadas pelo teste de comparação REGWQ a $5 \%$, usando o programa SAS Statistical Package (1995).

\section{RESULTADOS E DISCUSSÃO}

\section{Perdas de massa e de nutrientes pelo material vegetal incorporado ao solo nas parcelas de campo}

As perdas de massa e de nutrientes das bolsas de decomposição incorporadas ao solo nas parcelas de campo variaram relativamente pouco entre tratamentos, ao longo dos 82 dias em que as bolsas permaneceram no campo (Quadro 3). Nos primeiros 30 dias foram observadas perdas de aproximadamente 30 e $50 \%$ da massa seca e dos nutrientes, respectivamente. A partir desse período, as perdas foram menos intensas e mantiveram-se até o final do experimento. Na última data de coleta foram observadas perdas de massa e nutrientes significativamente menores nos dois tratamentos que continham esterco (E e CE) que nos dois que continham somente material vegetal ( $\mathrm{C} \mathrm{e} \mathrm{T).} \mathrm{As}$ massas restantes nos tratamentos E e CE, após os 82 dias, foram de 39 e $38 \%$, respectivamente, enquanto nos tratamentos C e T ficaram em 27 e $26 \%$, respectivamente, provavelmente devido à menor labilidade do esterco.

As perdas de $\mathrm{N}$ e $\mathrm{P}$ das bolsas tiveram dinâmica semelhante à das perdas de massa seca (Quadro 3). Nas primeiras datas de coleta, as perdas diferiram relativamente pouco entre os tratamentos, mas, no final do experimento, as perdas de nutrientes foram significativamente menores nos tratamentos E e CE que em $\mathrm{C} \mathrm{e} \mathrm{T}$, indicando que a decomposição do esterco acontece de forma mais lenta que a da biomassa de crotalária e das ervas espontâneas incorporadas ao solo. A decomposição mais lenta do esterco, quando comparada com a de adubos verdes e material vegetal, já foi relatada em diversos estudos em condições de clima tropical (Palm et al., 2001). Esses resultados dão suporte à hipótese de que a incorporação de esterco, apesar de adicionar mais nutrientes ao solo, não necessariamente leva à maior disponibilização de nutrientes para as plantas durante o ciclo de cultivo.

É interessante salientar que as perdas de K pela biomassa do material vegetal e esterco foram maiores que as de Ne P (Quadro 3). Provavelmente, isso ocorreu devido ao fato de o $\mathrm{K}$ não fazer parte de nenhum composto orgânico (Faquin, 1994). Dessa forma, o K fica prontamente disponível a partir do momento em que o material é incorporado ao solo e é iniciada a decomposição. Os resultados do presente estudo são compatíveis com os observados em Cottica et al. (1999), que avaliaram a decomposição e o teor de nutrientes na palha de aveia-preta ao longo do tempo e constataram que, passados 55 dias do manejo da cultura, o conteúdo de $\mathrm{K}$ da palha havia diminuído $92 \%$.

\section{Dinâmica da disponibilidade de nutrientes do solo nas parcelas de campo}

A incorporação de esterco e, ou, crotalária teve influência significativa na dinâmica da disponibilidade de nutrientes no solo das parcelas de campo ao longo do ciclo de cultivo da batata (Figura 2). Em amostras coletadas imediatamente após a incorporação dos adubos orgânicos e o plantio da batata, e também sete dias após o plantio (DAP), a concentração de $\mathrm{N}-\mathrm{NO}_{3}{ }^{-}$ $+\mathrm{N}-\mathrm{NH}_{4}{ }^{+}$(N mineral) no tratamento $\mathrm{E}$ foi significativamente menor do que nos tratamentos $\mathrm{Ce}$ $\mathrm{CE}$ e não diferiu daquela do tratamento T. Aos $7 \mathrm{DAP}$, a quantidade de $\mathrm{N}$ mineral no tratamento $\mathrm{CE}$ diminuiu em relação ao período anterior e tornou-se significativamente menor que no tratamento $\mathrm{C}$. Provavelmente, o $\mathrm{N}$ mineral no tratamento $\mathrm{C}$ foi maior no período inicial, devido à rápida decomposição da biomassa da crotalária, porém o $\mathrm{N}$ mineral do solo nesse tratamento diminuiu gradativamente ao longo do período de cultivo, à medida que a biomassa incorporada foi consumida. 
Quadro 3. Percentagem de matéria seca e nutrientes (N, P e K) remanescentes de adubos orgânicos contidos em bolsas de decomposição enterradas a $20 \mathrm{~cm}$ de profundidade, em um Neossolo Regolítico submetido a diferentes tratamentos de adubação orgânica durante o ciclo da cultura da batata

\begin{tabular}{|c|c|c|c|c|c|c|}
\hline \multirow{2}{*}{ Tratamento(1) } & \multicolumn{6}{|c|}{ Intervalo de coleta (dias após a colocação no campo) } \\
\hline & 7 & 22 & 37 & 52 & 67 & 82 \\
\hline & \multicolumn{6}{|c|}{ Massa seca (\%) } \\
\hline $\mathrm{C}$ & $78,4 \mathrm{a}^{(2)}$ & $58,8 \mathrm{a}$ & 36,9 a & $38,6 \mathrm{a}$ & $39,1 \mathrm{a}$ & $27,2 \mathrm{~b}$ \\
\hline $\mathrm{CE}$ & $72,2 \mathrm{ab}$ & $54,2 \mathrm{a}$ & $44,3 \mathrm{a}$ & $40,7 \mathrm{a}$ & $36,7 \mathrm{a}$ & $38,0 \mathrm{a}$ \\
\hline $\mathrm{E}$ & $59,3 \mathrm{~b}$ & $50,0 \mathrm{a}$ & 37,6 a & $41,3 \mathrm{a}$ & 39,8 a & $39,2 \mathrm{a}$ \\
\hline \multirow[t]{2}{*}{$\mathrm{T}$} & $70,43 \mathrm{ab}$ & $48,1 \mathrm{a}$ & 49,0 a & $42,7 \mathrm{a}$ & 39,2 a & $26,6 \mathrm{~b}$ \\
\hline & \multicolumn{6}{|c|}{$\mathrm{N}, \%$} \\
\hline $\mathrm{C}$ & $56,4 \mathrm{~b}$ & $57,2 \mathrm{a}$ & $20,6 \mathrm{~b}$ & $15,8 \mathrm{~b}$ & $17,9 \mathrm{a}$ & $17,7 \mathrm{~b}$ \\
\hline $\mathrm{CE}$ & 87,9 a & $57,5 \mathrm{a}$ & 49,5 a & $34,6 \mathrm{a}$ & 35,6 a & $42,9 \mathrm{a}$ \\
\hline $\mathrm{E}$ & $53,4 \mathrm{~b}$ & 36,8 a & $28,6 \mathrm{~b}$ & $31,4 \mathrm{ab}$ & $28,1 \mathrm{a}$ & $40,9 \mathrm{a}$ \\
\hline \multirow[t]{2}{*}{$\mathrm{T}$} & $48,9 \mathrm{~b}$ & 33,3 a & $34,0 \mathrm{ab}$ & $22,6 \mathrm{ab}$ & $31,0 \mathrm{a}$ & $24,5 \mathrm{ab}$ \\
\hline & \multicolumn{6}{|c|}{$\mathrm{P}, \%$} \\
\hline $\mathrm{C}$ & $47,5 \mathrm{a}$ & 38,9 a & $19,8 \mathrm{~b}$ & $13,1 \mathrm{~b}$ & $17,3 \mathrm{a}$ & $22,5 \mathrm{~b}$ \\
\hline $\mathrm{CE}$ & $52,6 \mathrm{a}$ & $33,1 \mathrm{a}$ & 31,9 a & $32,9 \mathrm{a}$ & $38,8 \mathrm{a}$ & $41,1 \mathrm{a}$ \\
\hline $\mathrm{E}$ & $48,8 \mathrm{a}$ & 36,9 a & 30,8 a & 32,5 a & $41,7 \mathrm{a}$ & 43,7 a \\
\hline \multirow[t]{2}{*}{$\mathrm{T}$} & 38,8 a & $33,8 \mathrm{a}$ & 29,8 a & $20,1 \mathrm{~b}$ & 28,8 a & $19,0 \mathrm{~b}$ \\
\hline & \multicolumn{6}{|c|}{$\mathrm{K}, \%$} \\
\hline $\mathrm{C}$ & $49,1 \mathrm{a}$ & $24,7 \mathrm{a}$ & $5,7 \mathrm{a}$ & $2,1 \mathrm{~b}$ & $1,9 \mathrm{a}$ & $1,6 \mathrm{~b}$ \\
\hline $\mathrm{CE}$ & $65,7 \mathrm{a}$ & $33,0 \mathrm{a}$ & $12,7 \mathrm{a}$ & $9,5 \mathrm{a}$ & 7,5 a & $7,4 \mathrm{a}$ \\
\hline $\mathrm{E}$ & $41,7 \mathrm{a}$ & $24,8 \mathrm{a}$ & $10,0 \mathrm{a}$ & $8,3 \mathrm{a}$ & $8,8 \mathrm{a}$ & $8,9 \mathrm{a}$ \\
\hline $\mathrm{T}$ & $54,3 \mathrm{a}$ & $22,5 \mathrm{a}$ & $14,3 \mathrm{a}$ & $5,9 \mathrm{ab}$ & $5,7 \mathrm{a}$ & $2,9 \mathrm{~b}$ \\
\hline
\end{tabular}

${ }^{(1)} \mathrm{C}=$ plantio e incorporação de Crotalaria juncea antes do plantio da batata; $\mathrm{E}=$ aplicação anual de $15 \mathrm{t}^{-1} \mathrm{de}^{-1}$ esterco de caprinos; $\mathrm{CE}=$ plantio e incorporação de $C$. juncea + aplicação anual de $7,5 \mathrm{t} \mathrm{ha}^{-1}$ de esterco; $\mathrm{T}=$ testemunha sem adubação. ${ }^{(2)}$ Médias na mesma coluna, para cada elemento, seguidas pela mesma letra não diferem pelo teste de comparação REGWQ $(\mathrm{P}<0,05)$.
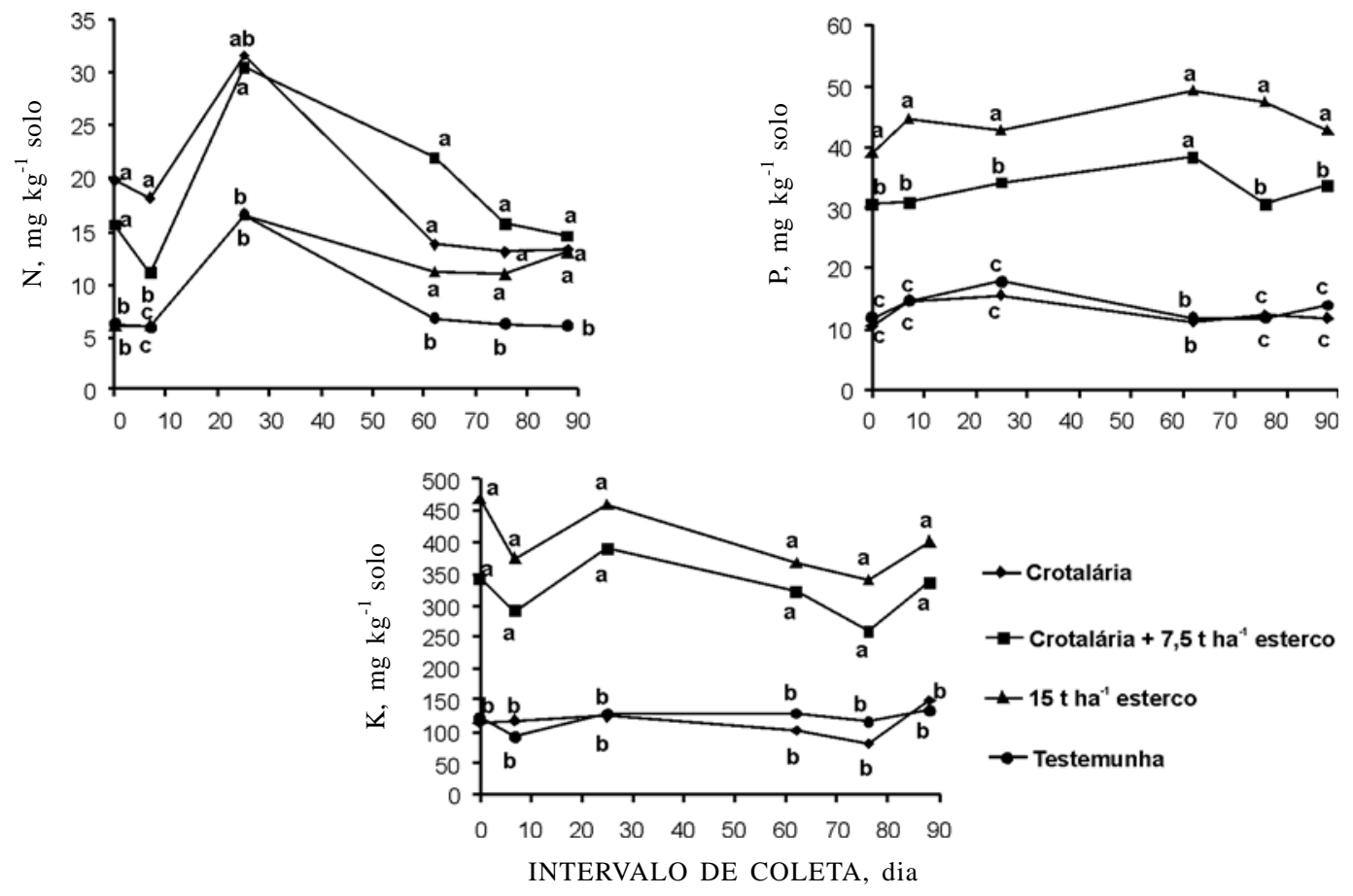

Figura 2. Teor de $\mathrm{N}$ mineral $\left(\mathrm{N}_{-} \mathrm{NO}_{3}{ }^{-}+\mathrm{N}-\mathrm{NH}_{4}{ }^{+}\right.$) e $\mathrm{P}$ e K extraíveis (Mehlich-1), após incorporação de esterco e, ou, crotalária, em um Neossolo Regolítico, durante o ciclo da cultura da batata. 
$\mathrm{O} \mathrm{N}$ mineral aumentou em todos os tratamentos na coleta aos 25 DAP, o que pode ser explicado pela maior precipitação pluvial observada nesse período (Figura 1). Aos 62 DAP, o N mineral no tratamento $\mathrm{C}$ não diferiu do $\mathrm{N}$ mineral nos tratamentos $\mathrm{E}$ e $\mathrm{CE}$. $\mathrm{Na}$ última data de coleta, aos 88 DAP, a disponibilidade de $\mathrm{N}$ mineral no solo não diferiu entre os tratamentos $\mathrm{C}, \mathrm{CE}$ e $\mathrm{E}$, os quais apresentaram significativamente mais $\mathrm{N}$ mineral que o tratamento $\mathrm{T}$ (Figura 2). Até os $62 \mathrm{DAP}$, a disponibilidade do $\mathrm{N}$ no solo para os tratamentos $\mathrm{E}$ e $\mathrm{T}$ não diferiu significativamente. É importante salientar que, no momento da incorporação dos adubos orgânicos, o solo do tratamento $\mathrm{E}$ apresentava o dobro do valor do teor do $\mathrm{N}$ total (Quadro 1). Provavelmente, a maior recalcitrância do esterco e, possivelmente, da biomassa vegetal das ervas espontâneas incorporadas ao solo neste tratamento inibiu o início da mineralização do $\mathrm{N}$ contido nesse material orgânico.

Quanto ao P extraível no solo, o maior teor foi verificada no tratamento $\mathrm{E}$, seguido pelo tratamento $\mathrm{CE}$ e, finalmente, pelos tratamentos $\mathrm{C}$ e T. O comportamento do K extraível foi semelhante ao do $\mathrm{P}$, sendo significativamente maior, ao longo de todas as datas de coleta, nos tratamentos $\mathrm{E}$ e CE que em C e T (Figura 2). A alta disponibilidade de $\mathrm{P}$ e $\mathrm{K}$ no solo dos tratamentos com incorporação de esterco deve-se ao alto teor desses nutrientes na biomassa do esterco incorporado.

Dos elementos analisados, o $\mathrm{N}$ foi o único que se apresentou como limitante no tratamento E, em relação ao tratamento $\mathrm{CE}$, no período inicial após a incorporação dos adubos orgânicos. Conforme era esperado, esses resultados indicam que a incorporação do esterco levou à imobilização de $\mathrm{N}$ nas primeiras semanas, e esse, provavelmente, foi o fator que limitou o crescimento e a produtividade da batata nas parcelas de campo ao longo dos anos anteriores ao do presente estudo (Silva et al., 2003). Nas parcelas em que foram incorporadas $7,5 \mathrm{t} \mathrm{ha}^{-1}$ de esterco combinado com a incorporação da crotalária, a disponibilidade de $\mathrm{N}$ proveniente da decomposição da biomassa da crotalária aparentemente foi suficiente para evitar a imobilização de $\mathrm{N}$ do solo e para suprir a demanda da batata. Em resumo, o tratamento $\mathrm{CE}$ foi aquele que proporcionou disponibilidade de nutrientes do solo mais balanceada, tanto pela incorporação de $\mathrm{N}$ lábil na biomassa da crotalária, o que preveniu a imobilização de $\mathrm{N}$ do solo, quanto pelo fornecimento de nutrientes adicionados com os $7,5 \mathrm{t} \mathrm{ha}^{-1}$ de esterco, principalmente o $\mathrm{P}$ e o $\mathrm{K}$, fato crucial para manter a fertilidade do solo e sustentar a produtividade de tubérculos de batata nessas parcelas.

Produção de matéria seca e absorção de nutrientes pelo capim-buffel em casa de vegetação

A incorporação de esterco, combinado ou não com crotalária, teve influência significativa na produção de matéria seca do capim-buffel durante os 300 dias de cultivo (Figura 3). No primeiro corte, 35 dias após o transplantio (DAT), o tratamento CE levou a uma produção de massa seca significativamente maior da parte aérea do buffel, seguido pelos tratamentos $\mathrm{E}$ e $\mathrm{C}$, os quais produziram mais massa seca que o tratamento T. Comportamento semelhante foi observado na acumulação de N, P e K na biomassa aérea (Figura 3). No segundo e terceiro cortes, a produção de matéria seca e o acúmulo de $\mathrm{N}, \mathrm{P}$ e K pelo buffel no tratamento $\mathrm{E}$ foram significativamente maiores que nos demais tratamentos. A partir do quarto corte não houve diferenças entre os tratamentos $\mathrm{E}$ e $\mathrm{CE}$, os quais produziram mais matéria seca e acumularam mais $\mathrm{N}, \mathrm{P}$ e $\mathrm{K}$ que os tratamentos C e T (Figura 3).

Comparando a eficiência dos estrumes curtido e biodigerido durante 38 dias, Sampaio et al. (1985) relataram a superioridade deste último quanto à produção de matéria seca e absorção de nutrientes pelo milheto. Comportamento semelhante foi
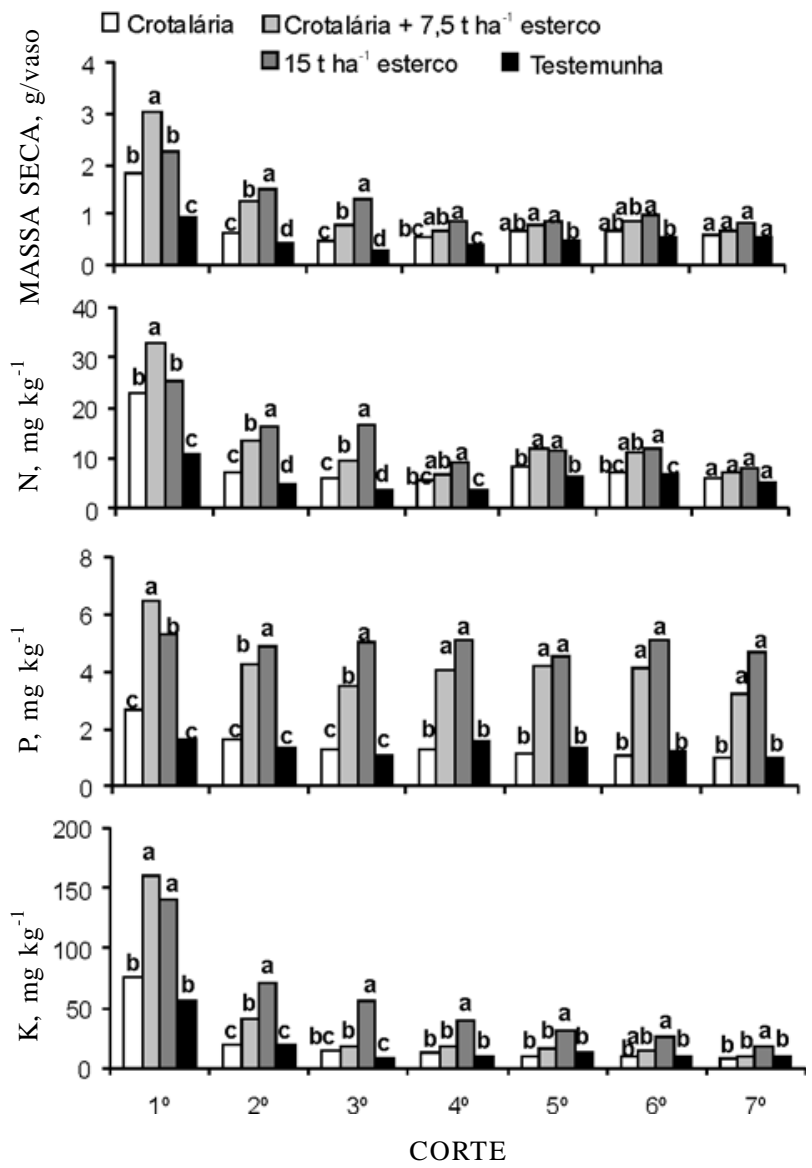

Figura 3. Produção de matéria seca e acúmulo de N, P e K em sete cortes sucessivos da parte aérea do capim-buffel cultivado por 300 dias em vasos com 700 g de solo (Neossolo Regolítico), após aplicação de esterco e, ou, crotalária. 
verificado, após 35 DAT do capim-buffel, no presente estudo, uma vez que o esterco incorporado ao solo já tinha sido parcialmente decomposto. Esses resultados são compatíveis com as demais observações deste estudo e suportam a hipótese de que a incorporação de esterco pode limitar o desenvolvimento vegetal no período inicial do ciclo de cultivo, devido à imobilização de N.

As maiores produções acumuladas de matéria seca do buffel aos 300 DAT foram de 10,2 e 9,7 g/vaso, nos tratamentos E e CE, respectivamente. Avaliando as produções de matéria seca do buffel, Fraga (2002) encontrou, após 60 dias de cultivo, produções que variaram entre 4,02 e 7,28 g/vaso.

A produção de matéria seca da raiz do capim-buffel aos $300 \mathrm{DAT}$ foi maior nos tratamentos $\mathrm{C}$ e $\mathrm{E}$, os quais não foram significativamente diferentes entre si, mas foram superiores aos tratamentos $\mathrm{CE}$ e T. O acúmulo de nutrientes $(\mathrm{N}, \mathrm{P}$ e K$)$ pelas raízes do capim-buffel não apresentou diferença significativa entre tratamentos, exceto para o $\mathrm{P}$, que foi maior no tratamento E em relação a C e T (Quadro 4).

Com o objetivo de avaliar o impacto potencial dos tratamentos estudados sobre a fertilidade do solo em longo prazo, foi calculado o balanço de nutrientes $(\mathrm{N}$, $\mathrm{P} \mathrm{e} \mathrm{K}$ ) no sistema solo-planta do experimento de casa de vegetação. A quantidade inicial de cada nutriente no sistema foi considerada igual à soma da quantidade da fração disponível do nutriente no solo (N mineral, $\mathrm{P}$ e K extraíveis) no início do ensaio e da quantidade do nutriente contido no esterco e, ou, material vegetal incorporado ao solo (Quadro 5). Dessa quantidade inicial foi subtraída tanto a quantidade total de nutrientes absorvida pelo capim-buffel nos sete cortes quanto a quantidade de nutrientes disponíveis no solo no final do ensaio.
Nos tratamentos em que foi aplicado esterco (E e $\mathrm{CE}$ ), o balanço de nutrientes indicou que a retirada destes pelo buffel foi menor que a quantidade incorporada. Esses tratamentos, portanto, levariam a aumento do teor de nutrientes do solo em longo prazo, o que é compatível com os resultados encontrados por Silva et al. (2005), em que se observou que o estoque de nutrientes do solo aumentou após cinco incorporações anuais de esterco seguidas do cultivo de batata. De forma semelhante, estudos em outras regiões do mundo relataram aumentos do teor de matéria orgânica do solo após a incorporação de adubos orgânicos de lenta decomposição por períodos prolongados (Palm, 2001).

No tratamento $\mathrm{C}$, a possível fixação biológica de $\mathrm{N}_{2}$ pela crotalária proporcionou a incorporação de biomassa lábil com baixa relação $\mathrm{C} / \mathrm{N}$, e isso provavelmente favoreceu a mineralização da matéria orgânica nativa do solo, proporcionando maior disponibilidade e absorção de nutrientes pelo buffel. Esse efeito, chamado de "priming", que depende do tipo de solo e do substrato orgânico adicionado (Alfaia, 1997), levou a balanços negativos de $P$, indicando que esse tratamento poderia levar à redução do estoque de $\mathrm{P}$ nativo do solo. A longo prazo, o aumento da mineralização de nutrientes do solo na forma orgânica, causada pela adição de substratos orgânicos lábeis, já foi relatado por outros autores (Hart et al.,1985; Azam et al., 1993; Ceretta et al., 2002).

A incorporação do material vegetal no tratamento $\mathrm{T}$ adicionou quantidade relativamente pequena de nutrientes ao solo e, como conseqüência, a retirada de nutrientes e o crescimento do buffel nesse tratamento também foram limitados. Esses resultados foram compatíveis com a produtividade de batata relatada por Silva et al. (2007) nas parcelas de campo desse experimento.

Quadro 4. Produção de massa seca e acumulação de nutrientes nas raízes de capim-buffel cultivado em vasos por 300 dias e submetido a sete cortes após a incorporação de esterco e, ou, crotalária, em um Neossolo Regolítico

\begin{tabular}{|c|c|c|c|c|}
\hline \multirow{2}{*}{ Tratamento(1) $^{(1)}$} & \multirow{2}{*}{ Produção de massa seca } & \multicolumn{3}{|c|}{ Acúmulo de nutriente } \\
\hline & & $\mathbf{N}$ & $\mathbf{P}$ & $\mathbf{K}$ \\
\hline \multicolumn{2}{|r|}{ g/vaso } & $\longrightarrow$ & $g^{-1}$ de & 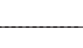 \\
\hline $\mathrm{C}$ & $1,9 \mathrm{a}^{(2)}$ & $18,5 \mathrm{a}$ & $1,6 \mathrm{~b}$ & $4,0 \mathrm{a}$ \\
\hline $\mathrm{CE}$ & $1,5 \mathrm{a}$ & $17,0 \mathrm{a}$ & $4,2 \mathrm{a}$ & $5,0 \mathrm{a}$ \\
\hline $\mathrm{E}$ & $1,8 \mathrm{a}$ & 19,3 a & $5,1 \mathrm{a}$ & $7,7 \mathrm{a}$ \\
\hline $\mathrm{T}$ & $1,0 \mathrm{a}$ & $9,8 \mathrm{a}$ & $1,1 \mathrm{~b}$ & $4,4 \mathrm{a}$ \\
\hline
\end{tabular}


Quadro 5. Balanço de nutrientes em vasos cultivados por 300 dias com capim-buffel, em um Neossolo Regolítico submetido a diferentes tratamentos de adubação orgânica

\begin{tabular}{|c|c|c|c|c|c|}
\hline \multirow[t]{3}{*}{ Nutriente } & Solo inicial ${ }^{(1)}$ & $\begin{array}{l}\text { Resíduo orgânico } \\
\text { incorporado }\end{array}$ & $\begin{array}{l}\text { Acumulado na } \\
\text { planta }\end{array}$ & Solo final ${ }^{(1)}$ & $\begin{array}{c}\text { Balanço de } \\
\text { nutrientes }\end{array}$ \\
\hline & & & $-\mathrm{mg} \mathrm{kg}^{-1}$ & 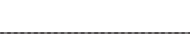 & + \\
\hline & \multicolumn{5}{|c|}{ Crotalária $^{(3)}$} \\
\hline $\mathrm{N}$ mineral & 32,1 & 42,0 & 66,8 & 6,1 & 1,2 \\
\hline P extraível (Mehlich-1) & 9,2 & 4,2 & 9,6 & 6,4 & $-2,6$ \\
\hline \multirow[t]{2}{*}{ K extraível } & 106,1 & 34,8 & 142,7 & 0,03 & $-1,8$ \\
\hline & \multicolumn{5}{|c|}{ Crotalária + esterco ${ }^{(4)}$} \\
\hline $\mathrm{N}$ mineral & 28,7 & 216,0 & 95,4 & 8,4 & 140,9 \\
\hline P extraível & 22,0 & 35,0 & 27,2 & 12,6 & 17,2 \\
\hline \multirow[t]{2}{*}{ K extraível } & 211,8 & 297,4 & 265,7 & 0,06 & 243,5 \\
\hline & \multicolumn{5}{|c|}{ Esterco $^{(5)}$} \\
\hline $\mathrm{N}$ mineral & 33,2 & 404,0 & 102,3 & 8,0 & 326,9 \\
\hline P extraível & 32,1 & 67,6 & 31,5 & 30,4 & 37,8 \\
\hline \multirow[t]{2}{*}{ K extraível } & 262,9 & 594,0 & 361,7 & 0,15 & 495,0 \\
\hline & \multicolumn{5}{|c|}{ Testemunha $^{(6)}$} \\
\hline $\mathrm{N}$ mineral & 20,9 & 27,0 & 40,8 & 4,7 & 2,4 \\
\hline P extraível & 10,0 & 4,5 & 8,3 & 4,6 & 1,6 \\
\hline K extraível & 194,2 & 44,9 & 115,5 & 0,05 & 123,6 \\
\hline
\end{tabular}

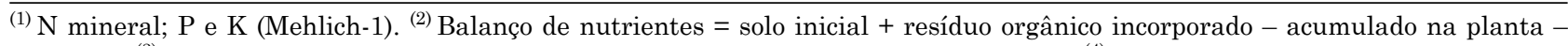
solo final. ${ }^{(3)}$ Plantio e incorporação de Crotalaria juncea antes do plantio da batata (C). ${ }^{(4)}$ Plantio e incorporação de $C$. juncea + aplicação anual de $7,5 \mathrm{t} \mathrm{ha}^{-1}$ de esterco (CE). ${ }^{(5)}$ Aplicação anual de $15 \mathrm{t} \mathrm{ha}^{-1}$ de esterco de caprinos (E). ${ }^{(6)}$ Testemunha sem adubação (T).

\section{CONCLUSÕES}

1. A incorporação de esterco na época do plantio, apesar de ter aumentado os teores de $\mathrm{P}$ e $\mathrm{K}$ do solo ao longo de todo o período de cultivo, causou imobilização de $\mathrm{N}$ do solo nas primeiras semanas após a incorporação.

2. A adubação verde com crotalária elevou a quantidade de $\mathrm{N}$ mineral do solo no período inicial de cultivo, mas não foi capaz de elevar os teores de $\mathrm{P} \mathrm{e} \mathrm{K}$ disponíveis no solo.

3. A combinação do plantio e incorporação da crotalária e a aplicação de esterco foi mais eficaz para disponibilizar nutrientes às plantas, pois foi capaz de evitar a imobilização de $\mathrm{N}$ do solo em curto prazo e elevou os teores de $\mathrm{P}$ e $\mathrm{K}$ disponíveis do solo ao longo do período de cultivo.

4. Recomenda-se que, em condições similares às do presente estudo, em que o esterco animal é de baixa qualidade e de decomposição lenta, no momento do plantio também seja adicionado um adubo orgânico rico em $\mathrm{N}$ e de rápida decomposição, como a biomassa de crotalária, para evitar a imobilização de $\mathrm{N}$ do solo durante o ciclo de cultivo.

\section{AGRADECIMENTOS}

Os autores agradecem o suporte técnico às atividades de campo e laboratório prestado por Clarindo C. Pontes, Claudenice E. Santos, Eduardo Medeiros, Gilberto E. do Nascimento, Ita Porto, Pedro A. da Silva Filho e Tadeu M. da Silva.

\section{LITERATURA CITADA}

ALFAIA, S.S. Mineralização do nitrogênio incorporado como material vegetal em três solos da Amazônia Central. R. Bras. Ci. Solo, 21:387-392, 1997.

ARAÚJO, A.P. \& ALMEIDA, D.L. Adubação verde associada a fosfato de rocha na cultura do milho. Pesq. Agropec. Bras., 28:245-251, 1993

AZAM, F.; SIMMONS, F.W. \& MULVANEY, R.L. Immobilization of ammonium and nitrate and their interaction with native $\mathrm{N}$ in three Ilinois Mollisols. Biol. Fertil. Soils, 15:50-54, 1993.

CERETTA, C.A.; BASSO, C.J.; HERBES, M.G.; POLLETTO, N. \& SILVEIRA, M.J. Produção e decomposição de fitomassa de plantas invernais de cobertura de solo e milho, sob diferentes manejos da adubação nitrogenada. Ci. Rural, 32:49-54, 2002. 
COTTICA, R.L.; PESSOA, A.C.S.; CRUSCIOL, C.A.C.; MARCON, E.; MORO, E. \& SOUZA, M.A. Persistência de cobertura morta e liberação de nutrientes da palhada de aveia preta em Latossolo Roxo cultivado com milho em plantio direto. CONGRESSO BRASILEIRO DE CIÊNCIA DO SOLO, 27., Brasília, 1999. Resumos. Brasília, 1999.

EMPRESA BRASILEIRA DE PESQUISA AGROPECUÁRIA EMBRAPA. Serviço Nacional de Levantamento e Conservação de Solos. Manual de métodos de análise de solos. 2 ed. Rio de Janeiro, 1997. 212p.

ENVIRONMENTAL PROTECTION AGENCY - EPA. Methods for chemical analysis of waters and wastes. Cincinnati, 1971. 312p.

FAQUIN, V. Nutrição mineral de plantas. Lavras, ESAL/ FAEPE, 1994. 227p.

FRAGA, V.S. Mudanças na matéria orgânica (C, N e P) de solos sob agricultura de subsistência. Recife, Universidade Federal de Pernambuco, 2002. 72p. (Tese de Doutorado)

HARMON, M.E.; NADELHOFFER, K.J. \& BLAIR, J.M. Measuring decomposition, nutrient turnover, and stores in plant litter. In: ROBERTSON, G.P.; COLEMAN, D.C.; BLEDSOE, C.S. \& SOLLINS, P., eds. Standard soil methods for long-term ecological research. New York, Oxford University Press, 1999. p.202-240.

HART, P.B.S.; RAYNER, J.H. \& JENKINSON, D.S. Influence of pool substitution on the interpretation of fertilizer experiments with ${ }^{15}$ N. J. Soil Sci., 37:389-403, 1986.

HOLANDA, J.S. Esterco de curral: composição, preservação e adubação. Natal, EMPARN, 1990. 69p. (Documentos, 17)

MENEZES, R.S.C.; SAMPAIO, E.V.S.B.; SILVEIRA, L.M.; TIESSEN, H. \& SALCEDO, I.H. Produção de batatinha com incorporação de esterco e/ou crotalária no Agreste paraibano. In: SILVEIRA, L.; PETERSEN, P. \& SABOURIN, E., orgs. Agricultura familiar e agroecologia no semi-árido: avanços a partir do agreste da Paraíba. Rio de Janeiro, AS-PTA, 2002. p.261-270.

MONEGAT, C. Plantas de cobertura do solo: características e manejo em pequenas propriedades. Chapecó, Ediçao do Autor, 1991. 337p.

MURPHY, J. \& RILLEY, J.P. A modified simple solution method for the determination of phosphate in natural waters. Anal. Chim. Acta, 27:31-36, 1962.
NASCIMENTO, J.T.; SILVA, I.F.; SANTIAGO, R.D. \& SILVA NETO, L.F. Efeito de leguminosas nas características químicas e matéria orgânica de um solo degradado. R.v. Bras. Eng. Agric. Amb., 7:457-462, 2003.

PALM, C.A.; GILLER, K.E.; MAFONGOYA, P.L. \& SWIFT, M.J. Management of organic matter in the tropics: translating theory into practice. Nutr. Cycling Agroecosy., 61:63-75, 2001.

ROSOLEM, C.A.; CALONEGO, J.C. \& FOLONI, J.S.S. Lixiviação de potássio da palha de espécies de cobertura de solo de acordo com a quantidade de chuva aplicada. R. Bras. Ci. Solo, 27:355-362, 2003.

SABOURIN, E.; SILVEIRA, L.M.; TONNEAU, J.P. \& SIDERSKY, P. Fertilidade e agricultura familiar no Agreste paraibano: um estudo sobre o manejo da biomassa. Esperança, CIRAD-TERA / ASPTA, 2000. 59p.

SAMPAIO, E.V.S.B.; SALCEDO, I.H.; ALVES, G.D. \& COLAÇO, W. Comparação entre estrume curtido e estrume biodigerido como fonte de nutrientes para o milheto. R. Bras. Ci. Solo, 9:27-31, 1985.

SAMPAIO, E.V.S.B.; SALCEDO, I.H.; SILVA, V.M. \& ALVES, G.D. Capacidade de suprimento de $\mathrm{N}$ e resposta à fertilização de 20 solos de Pernambuco. R. Bras. Ci. Solo, 20:269-279, 1995.

SAMPAIO, M.T. \& MALUF, W.R. Adubação verde: como contribuir para a saúde da horta, do homem e ainda obter lucro. Lavras, Universidade Federal de Lavras, Departamento de Agricultura, 1999. (Comunicado Técnico, 38)

SAS INSTITUTE. SAS Statistical Package, Version 6.12, Cary, 1995.

SILVA, T.O. Adubação orgânica da batata (Solanum tuberosum L.) com esterco e/ou Crotalaria juncea L. em um Neossolo Regolítico. Recife, Universidade Federal de Pernambuco, 2004. 47P. (Tese de Mestrado)

SILVA, T.O.; MENEZES, R.S.C.; TIESSEN, H.; SAMPAIO, E.V.S.B.; SALCEDO, I.H. \& SILVEIRA, L.M. Adubação orgânica da batata com esterco e, ou, Crotalaria juncea. I - Produtividade vegetal e estoque de nutrientes no solo em longo prazo. R. Bras. Ci. Solo. 31:39-49, 2007.

THOMAS, R.L.; SHEARRD, R.W. \& MOYER, J.R. Comparason of conventional and automated procedures for N, P and $\mathrm{K}$ analysis of plant material using a single digestion. Agron. J., 59:240-243, 1967.

TIESSEN, H.; CUEVAS, E. \& CHACON, P. The role of soil organic matter in sustaining soil fertility. Nature,371:783785,1994 